\title{
DECODING THE STAR OF BETHLEHEM
}

\author{
Sofias Sotirios* \\ *Corresponding Author: Sofias Sotirios, Geodesy (National Technical University of Athens, \\ Faculty of Surveying Engineering), Gis expert (ArcGIS ESRI), author of the books Bethlehem \\ Star Decoded, Orpheus \& Argonauts, Atlantis Of Plato, Centauri, Athens, Greece. \\ E-mail:-sotosofias@yahoo.gr
}

\section{ABSTRACT}

An analytical description of the astronomical phenomenon that was the "Bethlehem Star"

Keywords: Bethlehem, Magi, Saveh, Conjunction of Jupiter \& Saturn,

\section{INTRODUCTION}

The planetary conjunction of Jupiter and Saturn contains information that requires adept knowledge on Astronomy, which obviously the Zoroastrian priests possessed. The retrograde motion of the outer planets is observed at a point when the earth, moving at a higher angular speed, overtakes the outer planets and their motions create a loop, the shape of an $\mathrm{S}$ or a $\mathrm{Z}$ in the sky. This type of retrograde motion of the planets Mars, Jupiter and Saturn is visible to the naked eye and is known since antiquity. The retrograde motion of Mars is relatively easier to be observed as it lasts approximately two and a half months. Mars has a period of rotation of 687 days, is situated relatively near to the earth, and the arc drawn by its retrograde motion is of $15^{\circ}$ to $20^{\circ}$ degrees, easy to observe by sky connoisseurs. The retrograde motion of Jupiter lasts 11.86 years and that of Saturn last 29.46 years; due to their distance from the Sun they move relatively slow. Their retrograde motion draws an arc of $9^{\circ}$ and $6^{\circ}$, and it would take a highly skilled individual to distinguish with a naked eye their retrograde motion. It would take at least four months of continuous observation to study in full the retrograde motions of these two planets. The two giant planets conjunct approximately every 20 years, and it would be highly improbable to observe a simultaneous complete retrograde motion during the same period, particularly without running the risk of being interrupted when the planets enter daylight. The conjunction of Jupiter and Saturn occurred in the last century BC, twice, on the 9th of October $46 \mathrm{BC}$ and on the 30 th of June $26 \mathrm{BC}$. 

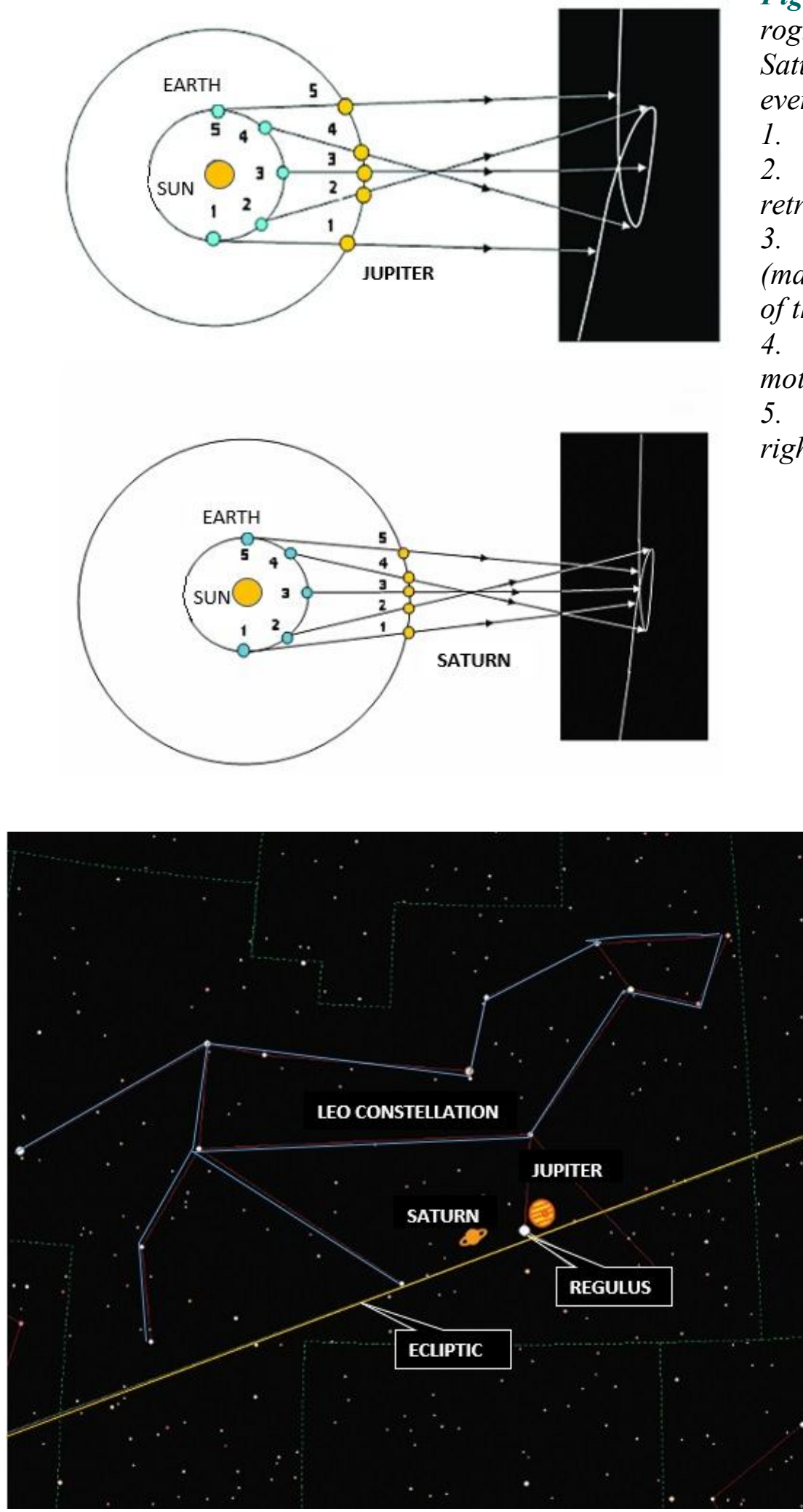

Figure 2. On the $19^{\text {th }}$ of November, 27 BC, something unique happened in the constellation of Leo. The retrograde motion of Jupiter and Saturn took place close to Regulus ( $\alpha$, Leo / $\alpha$ 'Leonis /Alpha Leonis) the brightest star of the Leo Constellation. This event helped the Magi to comprehend completely the retrograde motion of the two giant planets, since they used the "constant" Regulus as reference point.

After that date both planets resume their regular, motion and are getting prepared for their imminent conjunction. The conjunction of $26 \mathrm{BC}$ occurred in the constellation of Leo, on the $30^{\text {th }}$ of June, exactly next to Regulus A, also referred to as Cor Leonis (the Heart of the Lion). It was visible for an entire month on the western horizon before the planets faded away by the daylight. Previous researchers that had dwelled on the subject and the significance of Jupiter, Leo and Regulus were not aware of the retrograde planet motions in $27 \mathrm{BC}$ and of the conjunction that took place in $26 \mathrm{BC}$. The 
phenomena of the retrograde motion and conjunction I discovered and recorded for the first time in my research. This conclusively helped me decipher the Judaic tradition that considered Regulus and Leo as significant star and constellation respectively. Regulus manifested the Messiah and Leo the King. These two celestial objects demonstrated the concept of the retrograde motion to the ancient astronomers and prepared the Magi for the next great celestial rendezvous, the unique and unparalleled conjunction of $7 \mathrm{BC}$.

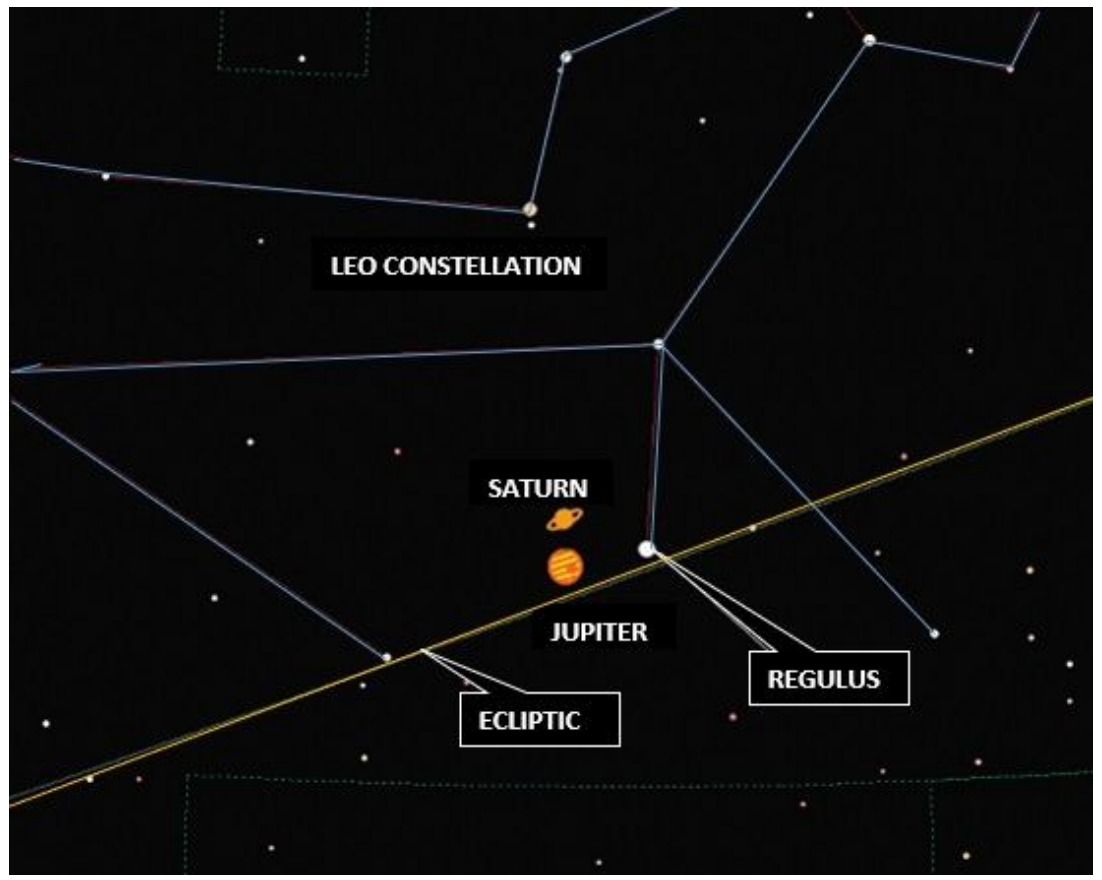

Figure 3. The conjunction of Jupiter and Saturn occurred on the 30th of June 26 BC, near the star Regulus (Latin) or Basiliscus (Greek) or Cor Leonis, a' Leonis. The total decoding and justification: Jupiter, the planet of Messiah according to the Jewish tradition, in conjunction with Saturn, the planet of the Jewish people, was hosted in the constellation of Leo, next to Regulus, the star of Messiah in the constellation of the King!! This must have been a clear sign for astrologers and mystics who could not overlook the great symbolism. Regulus helped the Magi to study the retrograde motion of the planets which occurred from November 27BC to March 26BC, thus preparing them for the very next conjunction of the planets, the unprecedented one in $7 \mathrm{BC}$.

Jupiter and Saturn conjunctions occur approx. every 20 years. Stored in the data base of Redshift 5 for the period 4700 B.C. to 10000 A.D. are 737 conjunctions. Triple conjunction, a conjunction where the planets in their motion cross paths three times, represent only $11 \%$ of the total, namely we have recorded only 83 triple conjunctions, with a non-periodical frequency of $40,80,120,140,160$, $220,260,300,340,380$, and 420 years.

In 7 B.C. we have the following triple consecutive conjunction:

- the first on June $4^{\text {th }}$, during the regular motion of the planets when Jupiter apparently reaches and overtakes Saturn.

- the second on September $23^{\text {rd }}$, when Saturn in an apparent retrograde motion reverses and overtakes Jupiter; and,

- The third on December $13^{\text {th }}$, when the right motion of Jupiter has commenced, and he seems to overtake Saturn for the last time. 


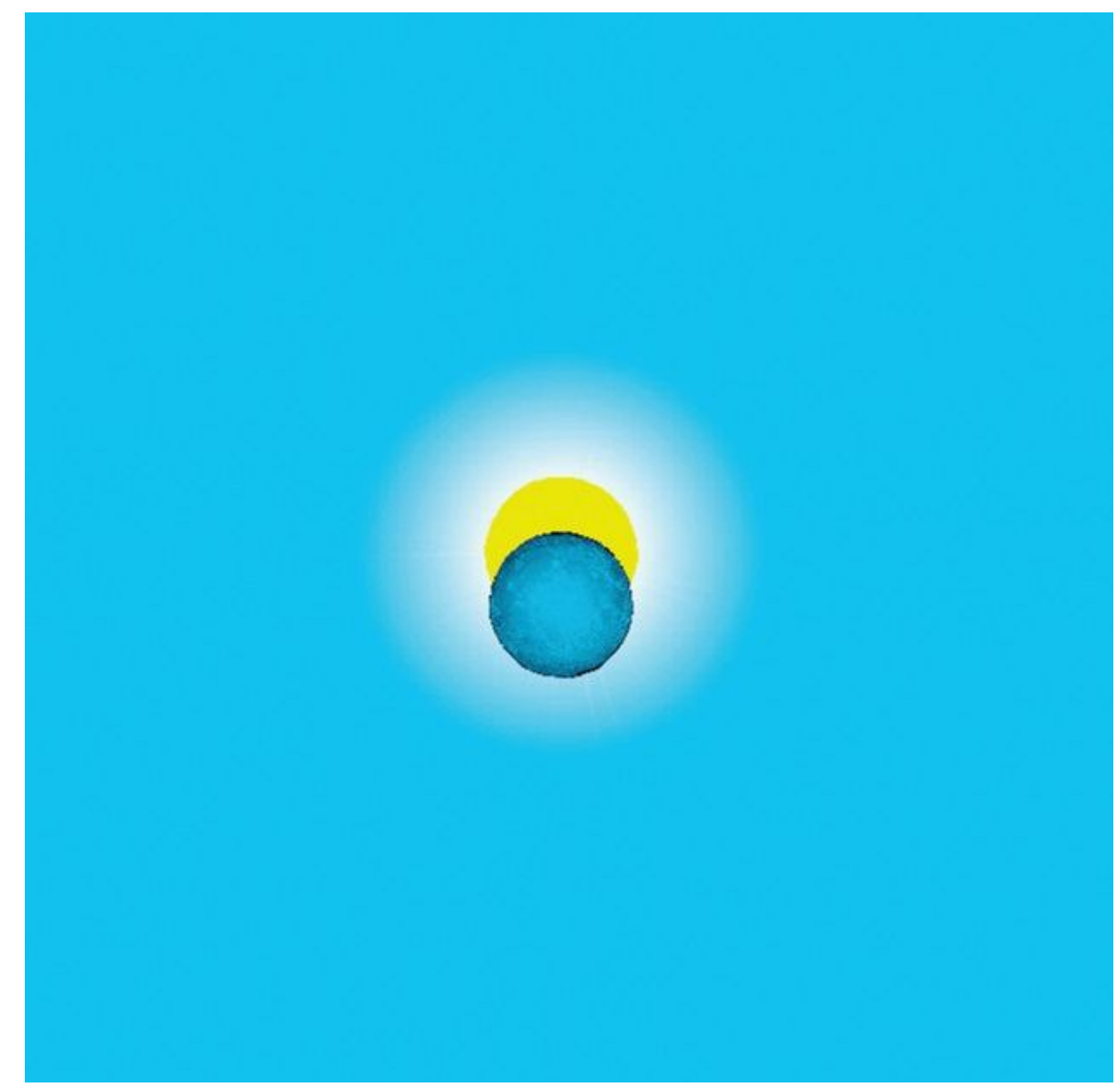

Figure 4. On the $29^{\text {th }}$ April, 7 B.C, at 17.38 p.m. the Magi of Persia admired a very good partial solar eclipse.

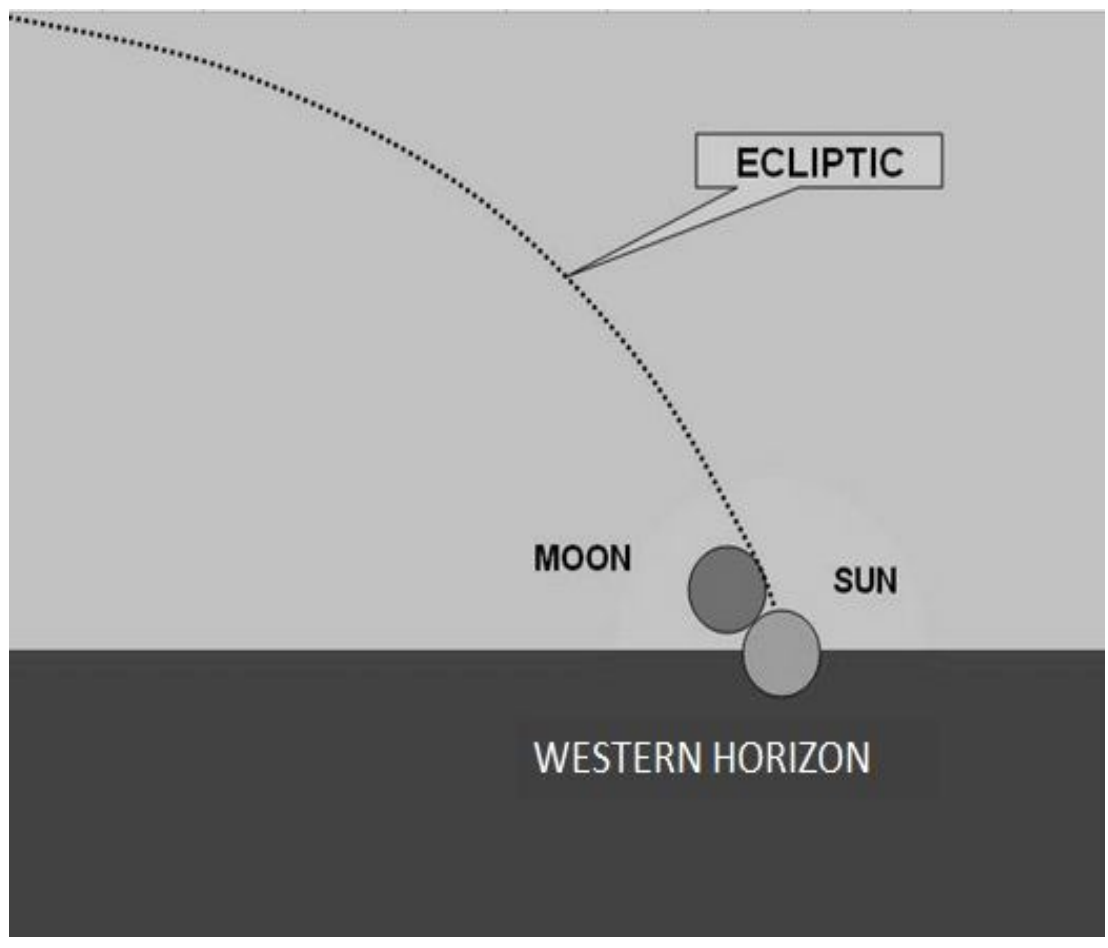

Figure 5. That day, the Sun rose at 5.17 a.m. and set at 18.42 p.m. local time.

The partial solar eclipseas a phenomenon- commenced at 16.38 p.m. and was completed at 18.42 p.m. Hence, exactly at 18.42 p.m. the solar and the lunar disks (adjacent photo) were osculating. This constitutes an amazing and rare phenomenon. Two disks, with the same apparent diameter, were in touch just before the sunset! 


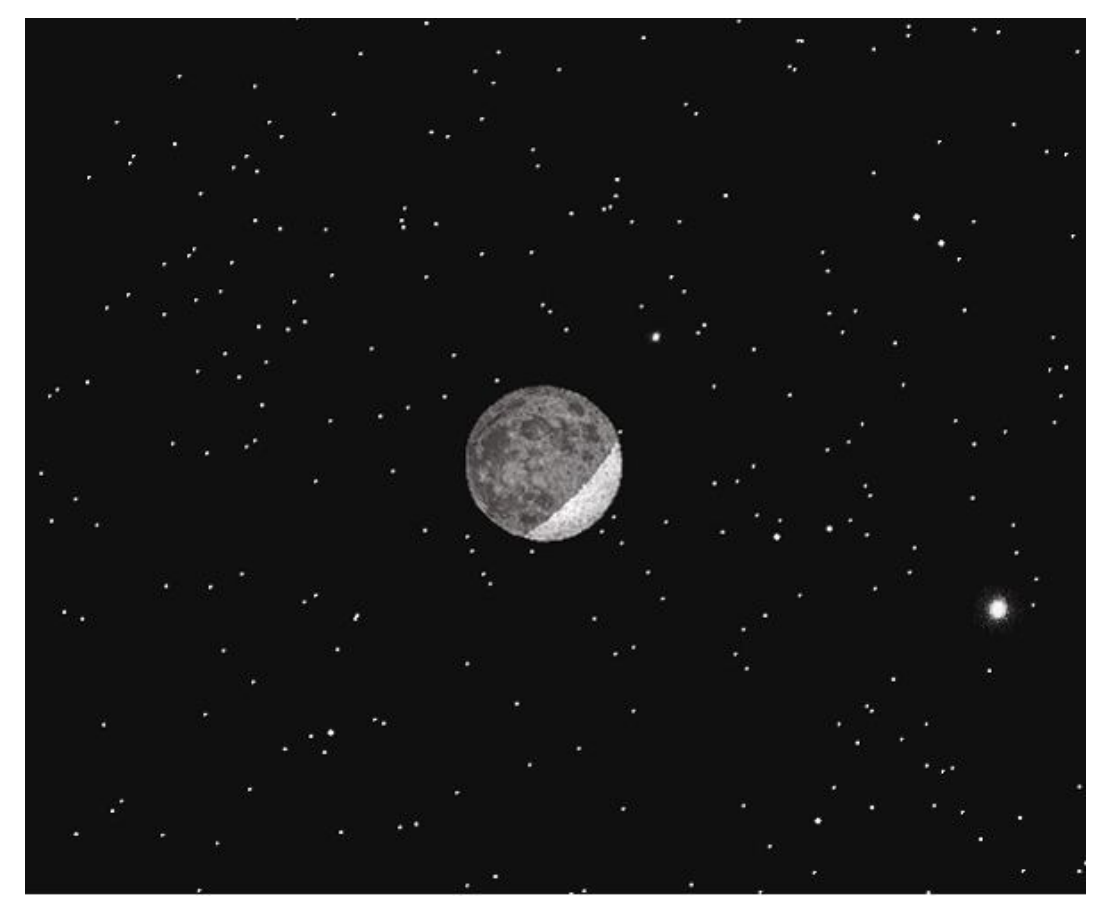

Figure 6. On the $13^{\text {th }}$ May, 7 BC, the Magi of Persia admired a partial lunar eclipse. It seems that in that unique year of $7 \mathrm{BC}$, all possible astronomical events took place almost simultaneously. An unusually busy year for astronomers!!

On the $4^{\text {th }}$ of June, 7 BC, Jupiter and Saturn align with the Earth and then the first conjunction occurs in the constellation of the Pisces. The planets are visible from

00:40 am till 04:20 am, just before the sunrise.

By calculating the sides of the celestial triangle formed, the results are distance from the Earth to the Sun 1.0175 AU, distance from the Earth to Jupiter5.071.017 AU and distance from Jupiter to the Sun 4.964.702 AU. If we apply to the above values the cosine $\left(\mathrm{a} 2=\mathrm{b} 2+\mathrm{c} 2-2^{*} \mathrm{~B}^{*} \cos \mathrm{A}\right)$ for the celestial triangle EarthSun-Jupiter, we find that angle A with vertex the Sun is $90^{\circ}$.

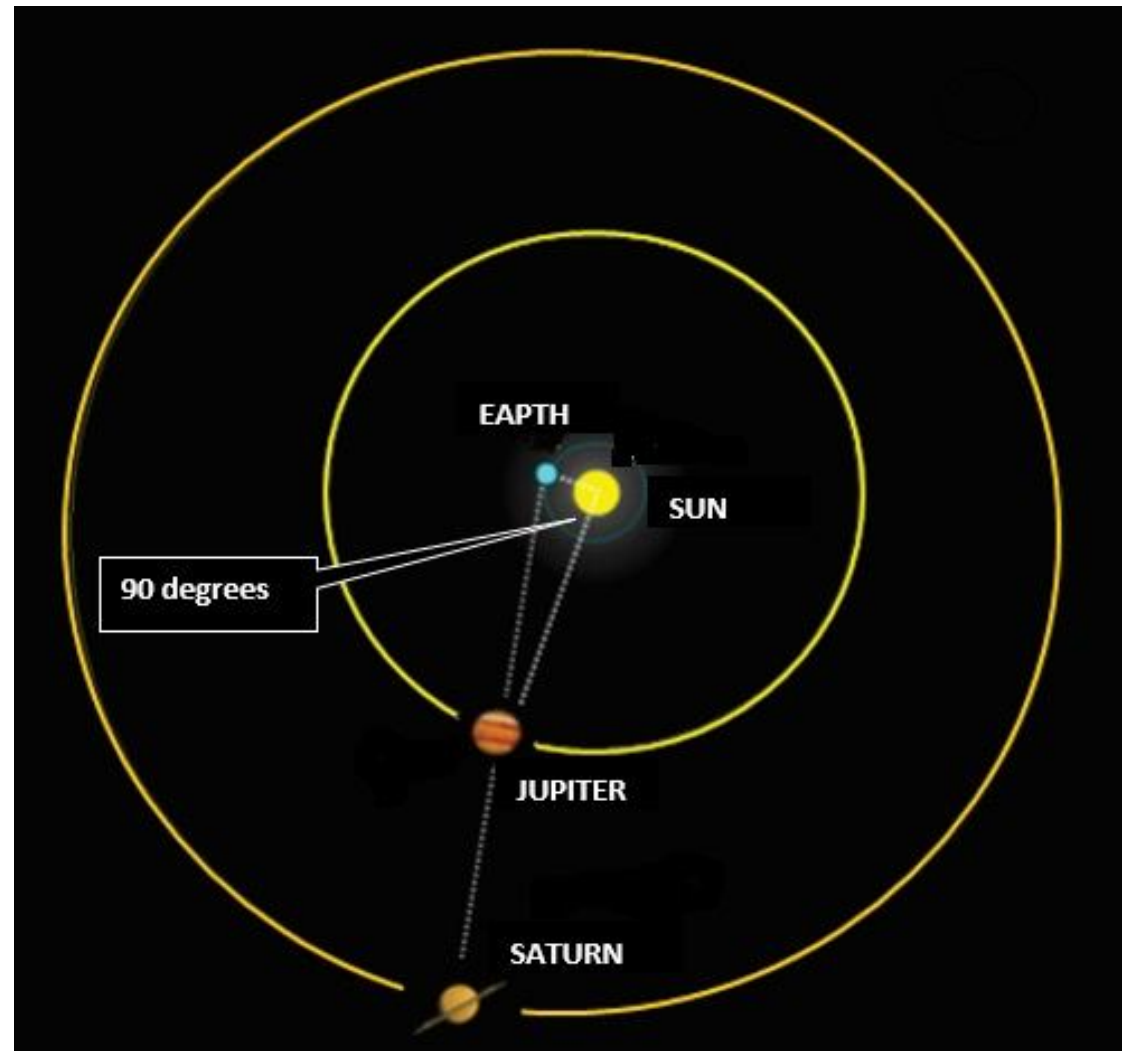

Figure 7. View plan of the solar system, during the first conjunction which occurred on the $4^{\text {th }}$ June, 7 BC. The angle with vertex the Sun (i.e. of the triangle, Earth, Sun, Jupiter) according to the planetary programs was at that time exactly $90^{\circ} ! ! !$

Jupiter and Saturn traveled together in a close unison for approximately a month. The conjunction is an event known to ancient astronomers, naturally the Magi as well, from previous occurrences. On the $8^{\text {th }}$ of July it appears that Saturn ceases to move in the sky, and thus starts its retrograde motion, it appears to move in the sky from the east to the west. On the same day Jupiter who is in regular motion for nine more days is positioned at a 
very particular point of its orbit. Its perihelion is at 4.964.391 AU from the Sun. On the $17^{\text {th }}$ of July Jupiter starts its retrograde motion and at that specific time the two planets appear to be moving from east to west, in relation to other stars situated further in the night sky.

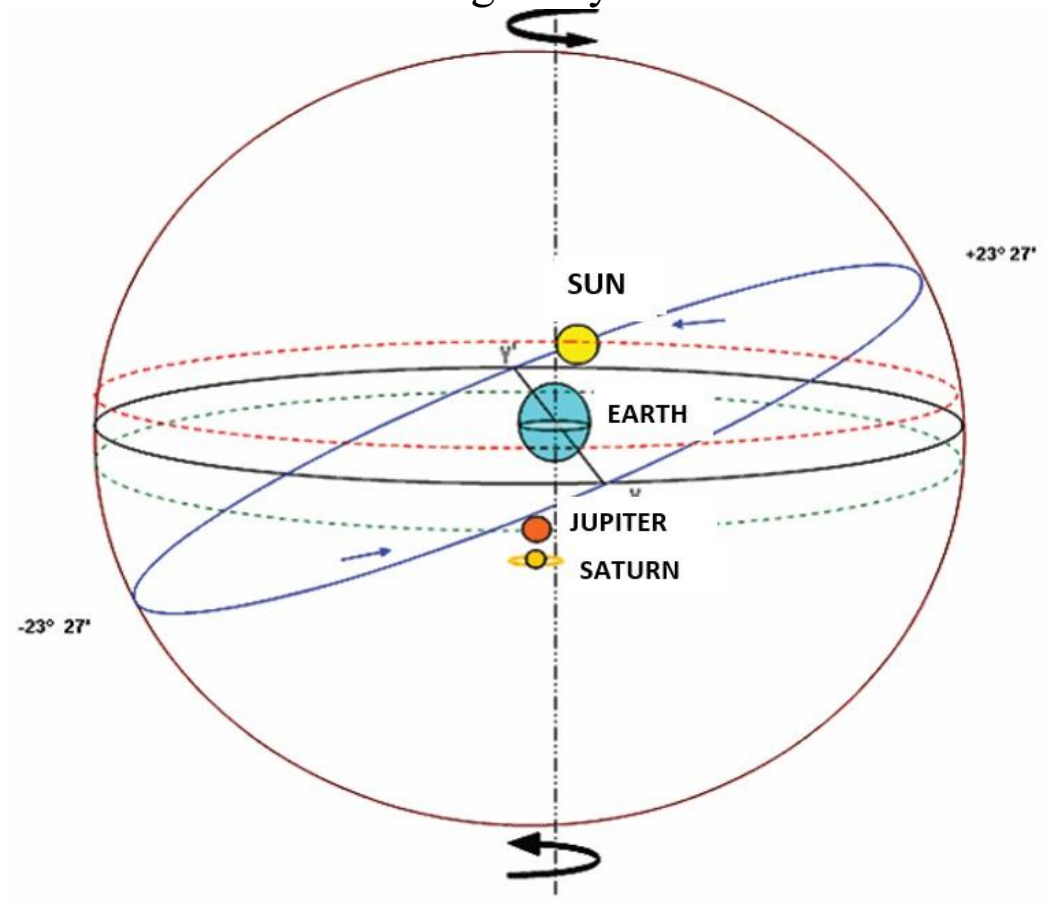

Figure 8. The apparent motion of the Sun and planets as recorded on the $14^{\text {th }}$ September $7 B C$. We can notice an astonishing heliocentric conjunction of Earth, Jupiter, and Saturn which gone unnoticed for almost 2012 years. The triple conjunction of Jupiter \& Saturn in 7 BC, was indeed quadruplicate, considering the participation of the Earth in the planetary pattern!!!

On the $14^{\text {th }}$ of September, $7 \mathrm{BC}$ the Perfect Great Conjunction, became unique, due to the absolute symmetry that it entailed. It marked the middle of the retrograde motion of both planets. Jupiter started its retrograde motion on the $17^{\text {th }}$ of July (the $198^{\text {th }}$ day of the year) and concluded it after 118 days, on the $12^{\text {th }}$ of November, the average point being $257(198+118 / 2=257)$. The $257^{\text {th }}$ day of the year is the $14^{\text {th }}$ of September. Saturn commenced its retrograde motion on the $8^{\text {th }}$ of July (the $189^{\text {th }}$ day of the year) and concluded it after 136 days, on the $21^{\text {st }}$ of November, the average point being again $257(189+136 / 2=257)$, same as before, the $14^{\text {th }}$ of September. On that date the distance from Jupiter to the Sun was 4.965.693 $\mathrm{AU}$, that is 4.965.693/4.964.391=1,00026 times the distance of the perihelion from the Sun.

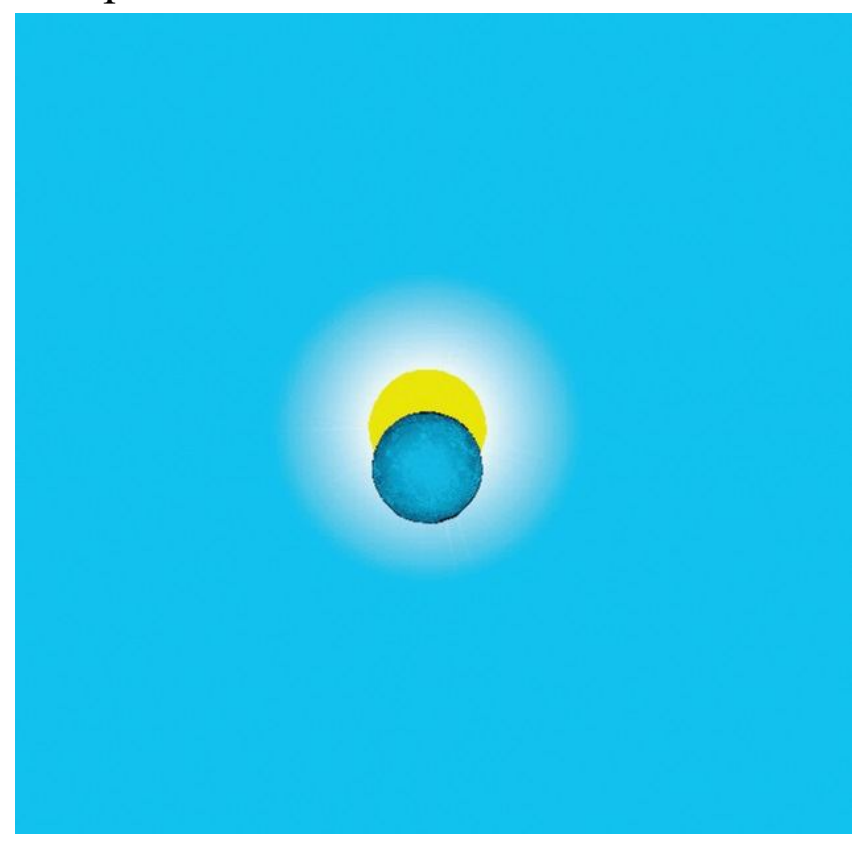

Figure 9. On the $23^{\text {rd }}$ October 7 BC, at 7.27 am, few days before the arrival of the Magi at Bethlehem, a second partial solar eclipse occurred within the same year. Just out of sheer curiosity, I searched in the data base of Redshift 5 for double annual solar eclipses, visible from Saveh and Jerusalem, over an extended period of 400 years (i.e. 200 before and 200 after Christ). Surprisingly, only in 7 BC two consecutive solar eclipses occurred in the same year!

On that same date the distance from Jupiter to the Earth was at its shortest point, at 3.969.549 AU and Earth was aligned to the Sun-Jupiter-Saturn. Jupiter was situated almost at 
the perihelion and exactly opposite the Earth, in a phenomenon similar to that of the full Moon, and with $99,999 \%$ of its surface illuminated by the Sun with an equatorial diameter of 49,6". This conjunctional Jupiter was at its brightest (with an apparent size of $-2,93$ ) and at exactly 05:23 am it revealed the country of the Magi's destination. Saturn was at 8.294.988 AU from the Sun, had also 99,999\% of its surface covered with sunlight, with an apparent equatorial diameter of 19, 9". On the $23^{\text {rd }}$ of September both planets were situated at the exact same point in the sky (Right Ascension=23h 20' 24'), which is an absolute conjunction, the second observed for this year. However, as the planets travel in very close proximity, we can safely conclude that for the period from 10 May to 31 December, the two planets appeared as in a continuous conjunction.

By the end of their retrograde motion, it appeared as if the planets decelerated, almost stopped completely their angular velocity. On the $12^{\text {th }}$ of November, $7 \mathrm{BC}$ the giant Jupiter appears to be motionless in the sky, in relation to other distant stars, while Saturn as its retrograde motion is coming to an end, also appears stationary. The wise and adept astronomers, the Magi, they observed two out of the five planets fixed in the sky, the Gospels refer to the Star of Bethlehem remaining still in the night sky, that was indeed the day the Messiah was born, when Jupiter and its traveling companion Saturn appeared to have stopped, motionless, stationary, an extraordinary celestial phenomenon.

SIMULATION OF THE NIGHT SKY ON 12 NOVEMBER 7 B.C. The Star of Bethlehem: The Great Conjunction of Jupiter \& Saturn

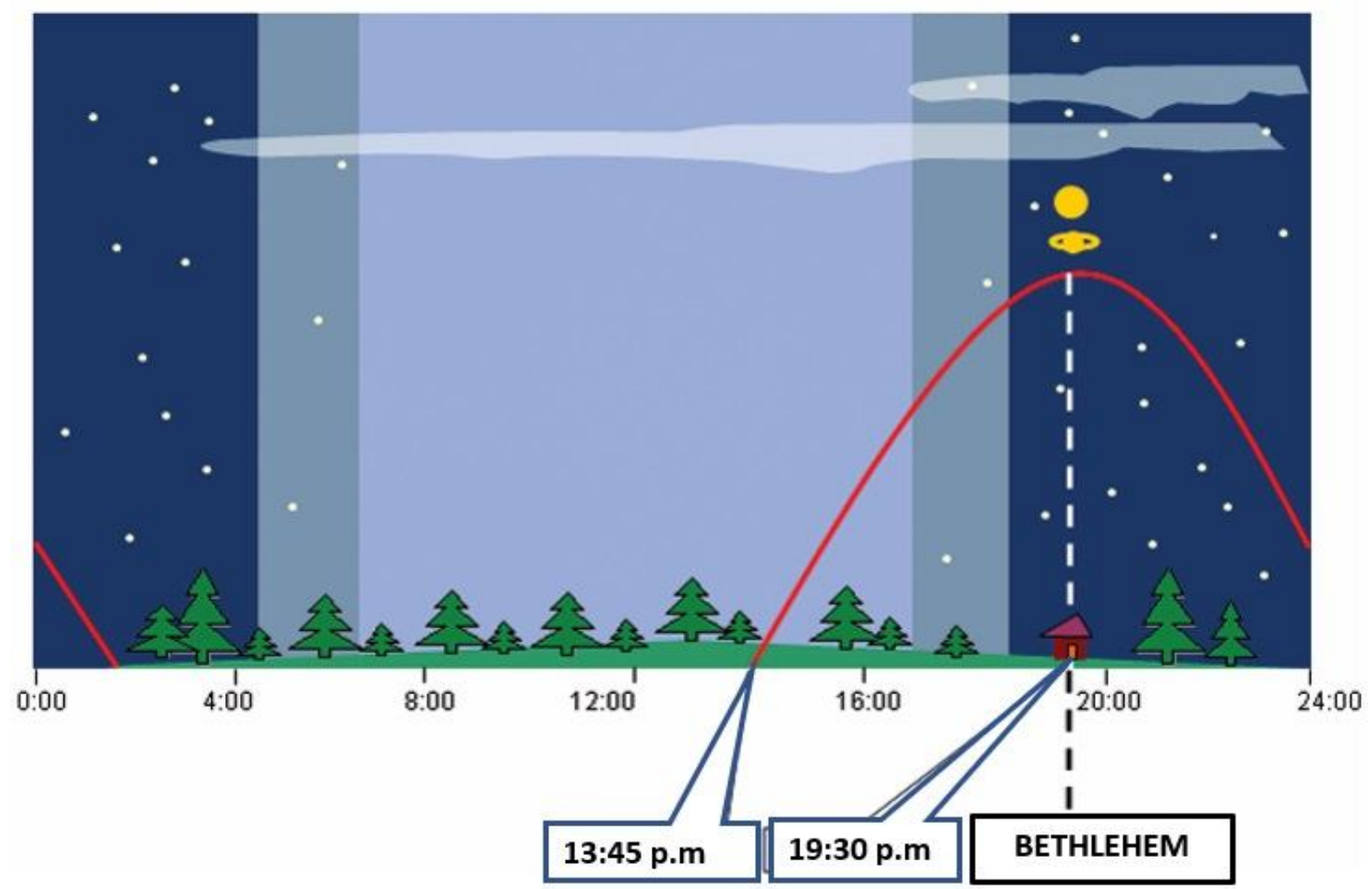

Figure 10. The above illustrates the phenomenon referred to by the Gospels as the "motionless star" over the Manger in Bethlehem. This is its scientific explanation: The end of the retrograde motion of both Jupiter-Saturn and the simultaneous crossing of the Bethlehem's meridian at 19:30 pm, on the $12^{\text {th }}$ November, $7 \mathrm{BC}$. 


\section{TWENTY CENTURIES OF QUESTIONS THAT REMAINED UNAN- SWERED CAN NOW BE ADDRESSED}

- The Star of Bethlehem, the conjunction of Jupiter with Saturn in 7 B.C. was it visible only to the Magi? Negative. It was visible to all the people, but few possessed the knowledge of celestial observation. Additionally, the uniqueness of the conjunction lies in its apparent duration, a whole year, and not so much in the brightness of the stars involved. Additionally, adept stargazers like the Magi, were not many, and few could interpret the complex patterns of the celestial vault, but for those few the conjunction of $7 \mathrm{BC}$ was astonishing and of unprecedented complexity. The Magi, with their knowledge of the Scriptures and the prophecies they interpreted the celestial event correctly and were able to witness the birth of the Messiah. For the peasants in Judea or in Persia two bright stars in the early morning sky in March, 7 BC did not signify anything important. But they were not aware of the planets, their motions, and their conjunctions. This knowledge was limited to few Greek astronomers, maybe some Egyptians priests, Mesopotamian astrologers, and few Chinese.

Even today, with an abundance of knowledge and resources, few can point to the planets in the sky. Even fewer can find Saturn among the many brighter stars that adorn the skies. The retrograde motions of the planets, the cause behind this type of motion to inner and outer planets are secrets hidden from the vast public. Even more so 2000 years ago, people concerned with their daily lives, had little time to waste gazing at odd celestial phenomena that they were not even aware of.

- Did the Star guide the Magi? Yes, it did. It pointed to their destination, which was known to them ahead of time, the city of Bethlehem in Judea. They took the well threaded route of the Silk Road that started in China, traversed Persia, and ended at the Mediterranean ports. The must have joined a caravan traveling west, and safely completed their journey. The knowledge they possessed guided them, their correct interpretation of the celestial events that were fast developing in front of their expert eyes. They were adept star gazers; they could distinguish the ecliptic plane in the night sky that marks the east and west points in the horizon. The east point where each night one by one the zodiac constellations appear and the west point to where they disappear. Moreover, the polar star, at that period known as one of the two "Guardians of the North", known by its Arabic name Kochab (northern star in Arabic), the b' of the Ursa Minor, steadily and reliably pointed the direction of the geographical North. They had tools to assist them, planetary maps, astrolabes, sextants, hourglasses and possibly sundials all carefully studied to provide directional guidance to the expert user.

- Did the Star stand still over the Manger? Not in a conventional interpretation. There is no celestial phenomenon that remains still in the sky, as the earth moves from west to east the sky also moves in a retrograde direction from east to west. 
Subsequently, all the celestial bodies appear, rise and fall in the sky and none remains inert for more that few seconds in a day. The star of Bethlehem, as so far explained was a natural phenomenon, unique but natural, and as such it followed the laws that pertain to motion. It did not stand still over the manger; it remained in the meridian of Bethlehem enough to mark the significance of the event taking place. A longer stay, beyond the norm and the natural, would have been an unnecessary and dangerous pole of attention of the birth of the Messiah at such perilous times.

- What was their descent? In the epic of Marco Polo describing his travels in Asia, there is a remarkable reference to the Magi from the inhabitants of a small Persian town known as Saveh. The town is situated $125 \mathrm{kms}$ southwest of Tehran and when Marco Polo passed through there on his way to China in 1271 AD, the inhabitants with exemplary pride showed him the burial grounds of the Magi. The three sanctified bodies were kept well preserved with their hair and beards adorning their faces, in elaborate mausoleums, almost intact after the passage of thirteen centuries. The city of Saveh was also known in antiquity as a center of astronomical studies, like a university, that had remained active till the city was destroyed by Chinggis Khan. That would explain the knowledge the Magi possessed.

Based on historical records, the Moguls under Chinggis Khan occupied and destroyed central and northern Persia in 1221 AD. The town of Saveh did not escape the rage of the invader; they destroyed the local Observatory, but the infamous Chinggis Khan obviously respected the mausoleum where the three Magi were buried, otherwise Marco Polo's account 50 years later would not stand.

I will further endeavor to prove that Saveh was the place of descent of the Magi applying the scientific skills of geodesy. This region of Persia is situated at $\varphi=35^{\circ}$ latitude, an unexpected divine gift; it has only a small difference in latitude from Bethlehem situated at $\varphi=31,7^{\circ}$. The Magi traveling westwards at approximately the $32^{\circ}$ latitude would see the same sky they had studied and closely observed in their hometown. All planetary motions would be familiar to them and as recorded in their books, and they could calculate accurately the positions of Jupiter and Saturn in relation to other known celestial bodies. In astronomy the definition of a star's or a planet's position is measured in horizontal and uranographic coordinates.

Horizontal coordinates are the Azimuth and the Altitude. Uranographic coordinates are the Right Ascension (R.A.) and the Declination. The method most used to define the position of celestial bodies is that of the Horizontal Coordinates, due to its simplicity. Azimuth is the angle on the horizontal level (or the horizon of a place), which begins from the point of cross section of the vertical level - that contains the zenith of a place and the geographic north - with the horizontal level and with a clockwise movement, from east to west. On the $14^{\text {th }}$ of September, 7 $\mathrm{BC}$, at the time Jupiter was setting at 05:28am its astronomical azimuth was $\mathbf{2 6 2}^{\mathbf{}}$, based on the data provided by the relevant computer program.

Similarly, the geodetic azimuth of Bethlehem, for the observer from Saveh, is 
calculated by deducting from the complete angle of 360deg the internal angle of the triangle. . The analytical calculation is here:

https://pdfhost.io/v/mPlBdZci. saveh

I have calculated the Azimuth (Saveh-Bethlehem) as $360^{\mathrm{O}}-98,16^{\mathrm{O}}=261,84^{\mathrm{O}}=$ $\sim 262^{\mathrm{O}}$.

Amazingly we conclude that Jupiter's azimuth is exactly the same as the geodetic azimuth of Saveh-Bethlehem!!

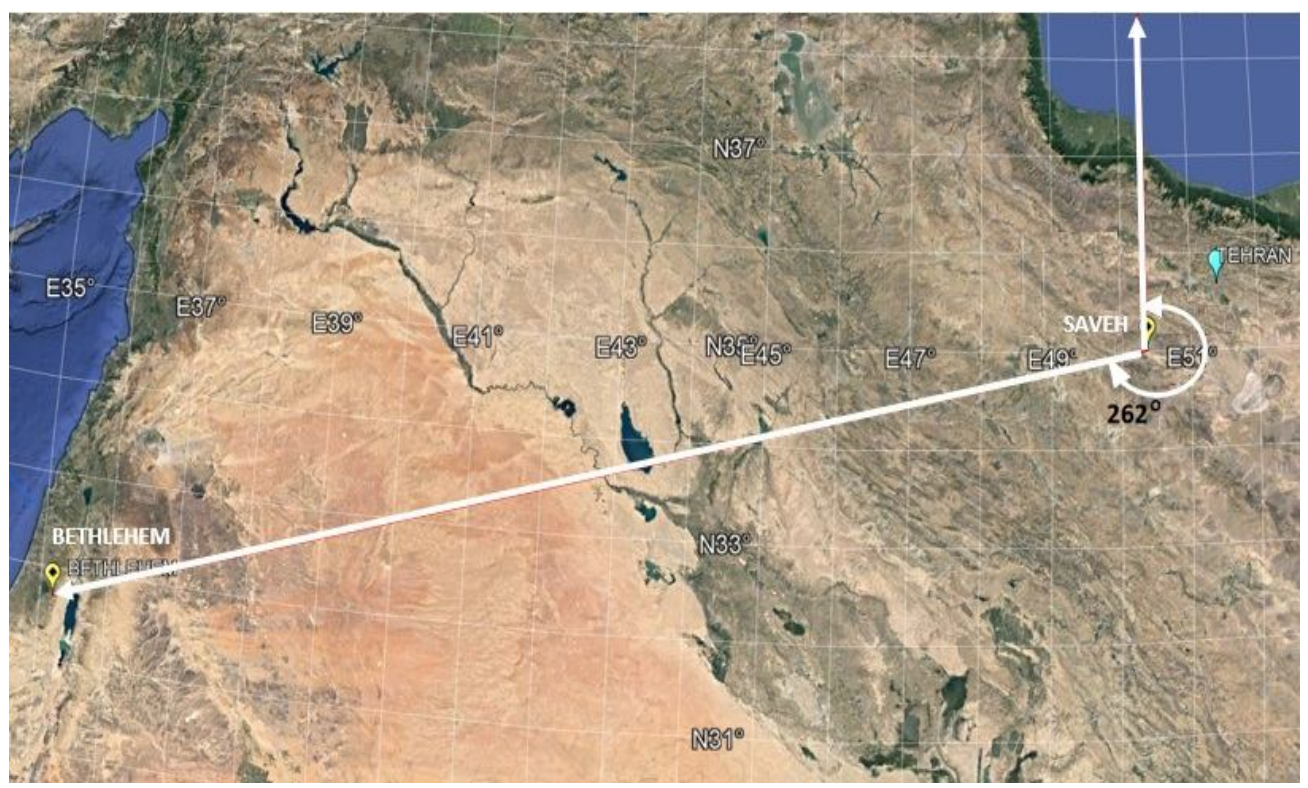

Figure 11. In the first photo, the azimuth of Saveh on the global maps is constant and it is always $262^{\circ}$.

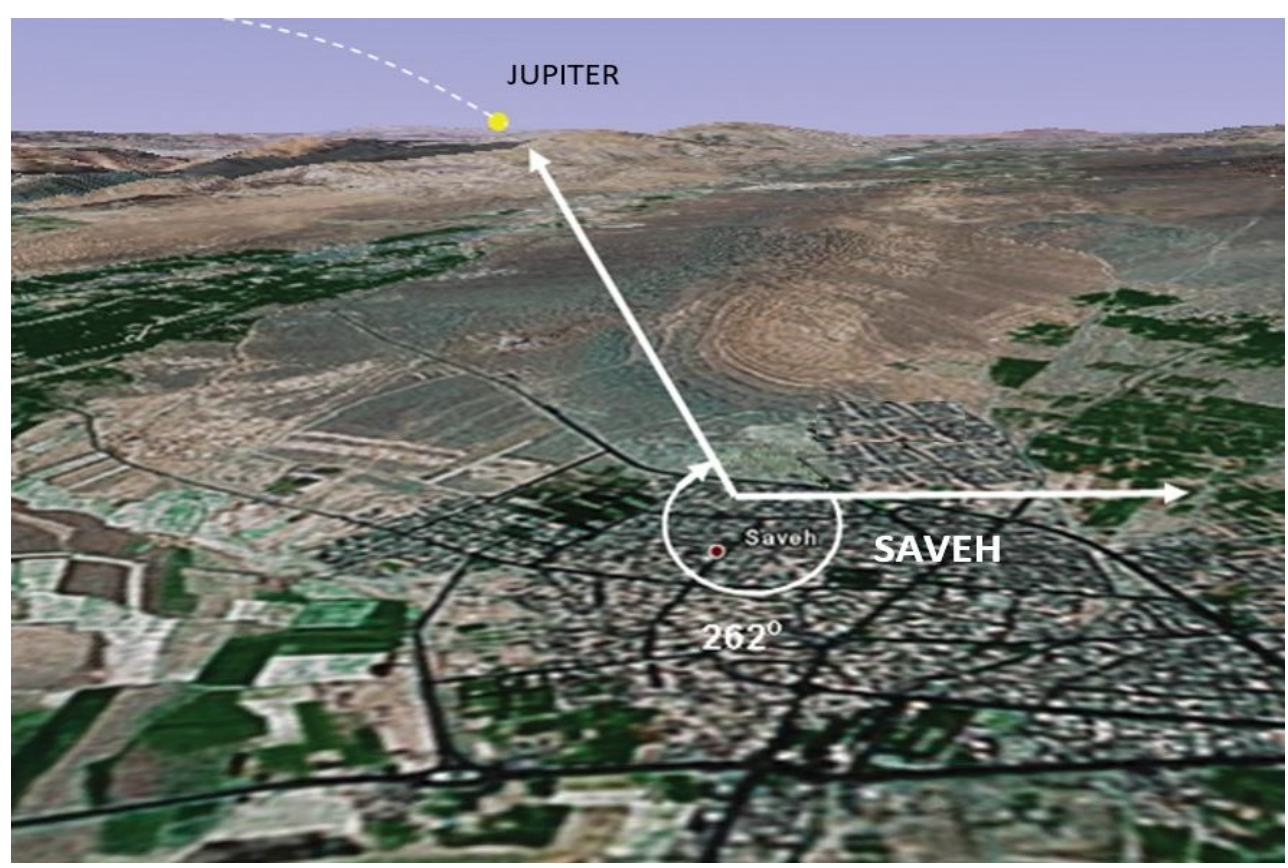

Figure 12. In the second photo, a random resident of Saveh should see, on the 14th September, 7 $B C$, just before the dawn, only the planet Jupiter (at its greatest brightness with apparent magnitude $m=$ 2,93) ready to set (i.e. Saturn with apparent magnitude $m=0, \quad$ should have been disappeared from sight earlier by daylight). The azimuth of Jupiter, according to the astronomical planetary programs, was at that time, $262^{\circ}$. An incredible "coincidence" indeed!

At 05:28am when the planet was setting at the western horizon it was situated at the shortest possible distance from the earth. At that exact position it points to the Magi their destination. It is the first day in a 5-month period when the setting of the planet, at least one of the two, is visible. By scientific calculations we 
rendered further evidence that Saveh was indeed the place from where the Magi started their journey, their place of descent. Marco Polo's account was not fictional, he documented historical facts.

\section{PERIOD OF THE PHENOMENON}

To calculate the period of the phenomenon we need not only the three orbital periods of Earth, Jupiter \& Saturn but the synodic periods of the planets as well. The synodic period of an outer planet to the Earth is calculated by Copernicus' formula: $\mathbf{1} /$ T PLanet $=\mathbf{1} / \mathbf{T}$ Earth $-\mathbf{1} / \mathbf{S i}$, where $\mathrm{T}=$ orbiting period around the Sun, and $\mathrm{Si}=$ the synodic period. This formula can be applied to the Jupiter - Saturn pair, as Saturn is an outer planet in relation to Jupiter. For our calculation we shall consider three synodic periods, one for each pair of planets. Applying the above formula, the results have as follows:

- Jupiter - Saturn 19,8650 years.

- $\quad$ Earth - Jupiter 1,0920 years.

- $\quad$ Earth - Saturn 1,0351 years.

These synodic periods are not constant as they contain decimal number of rotations of the planets, in contrast to their orbits around the Sun which is an integral number. It also varies depending on the planet's position in its orbit. The synodic period of the Earth with Jupiter or Saturn has short width of fluctuation, as the orbit of the Earth has small eccentricity $(\varepsilon=0,0167)$, whereas the synodic period of the pair Jupiter - Saturn where their orbits are of greater eccentricity $(0,0484$ and 0,0557 respectively) it fluctuates from 18,9 to 20,6 years (see relevant Table at the Annex of Tables), as the planets velocity varies depending on its position, near the perihelion, the aphelion or other point of its orbit $\left(2^{\text {nd }} \mathrm{Law}\right.$ of Kepler).Thus we have a Common Rotation Time, the least of the common multiple of the six periods: $1,000 \times 11,8626 \times 29,4475 \times 19,8626 \times 1,0351 \times$ 1,0920. Considering the acceptable fluctuation of the synodic period of Jupiter Saturn, the result is $\sim 7840-7890$ years; hence we are looking for conjunctions of the two planets that would occur during that period. Redshift 5 came to the assistance, reliable as ever. From its database it retrieved a triple, heliocentric conjunction, fulfilling all the criteria of the $7 \mathrm{BC}$ conjunction, which is due to occur in 7879AD (i.e. its period :7 $\mathrm{BC}-7879 \mathrm{AD}=7885$ years).

\section{THE UNIQUE CHARACTERISTICS OF THE 7 BC CONJUNCTION}

Certain characteristics of the Perfect Great Conjunction cannot be found cumulatively in other conjunctions in the known history of the solar system, such as:

- As a triple conjunction it represents only $11 \%$ of the Jupiter-Saturn conjunctions.

- It occurred in the constellation of the Pisces that hosted at the era of Jesus' birth the vernal point $(\gamma)$ gamma. This distinctive point every 2000 years is situated in a different zodiac constellation.

- The first one of the triple conjunction phenomenon formed a triangle Earth-SunJupiter with the Sun as its vertex having an angle of exactly 90deg. 
- It occurred while Jupiter was situated at its perihelion, the most significant point of a planet's orbit.

- Saturn and Jupiter on the $14^{\text {th }}$ of September, were simultaneously found in opposition with the Earth, as a result they formed a rare heliocentric conjunction (Earth-Jupiter-Saturn).

- On the same date at the moment the Sun rose, the twin Jupiter-Saturn was setting, thus revealing to the Magi their point of destination.

- The conjunction was visible for an entire year, uninterrupted from daylight; it is more common to have conjunctions gradually vanished due to the intervention of the sunlight. It appeared in the early morning sky and after 10-11months when the Sun was approaching to hide them in the afternoon light, the planets had already moved eastward, due to their self-motion, thus extending the period of their visibility to almost a year! In conclusion this conjunction was observable for the maximum possible period expected for the visibility of the phenomenon.

- The planets were moving in proximity, resulting in an apparent continuous conjunction for almost ten months.

- The triple conjunction that will occur in $7879 \mathrm{BC}$ will be the immediate proceeding conjunction like that of $7 \mathrm{BC}$.

\section{THE GRAPH OF THE OSCILLATION OF THE PERIOD OF A SIMPLE CONJUNCTION OF JUPITER-SATURN}

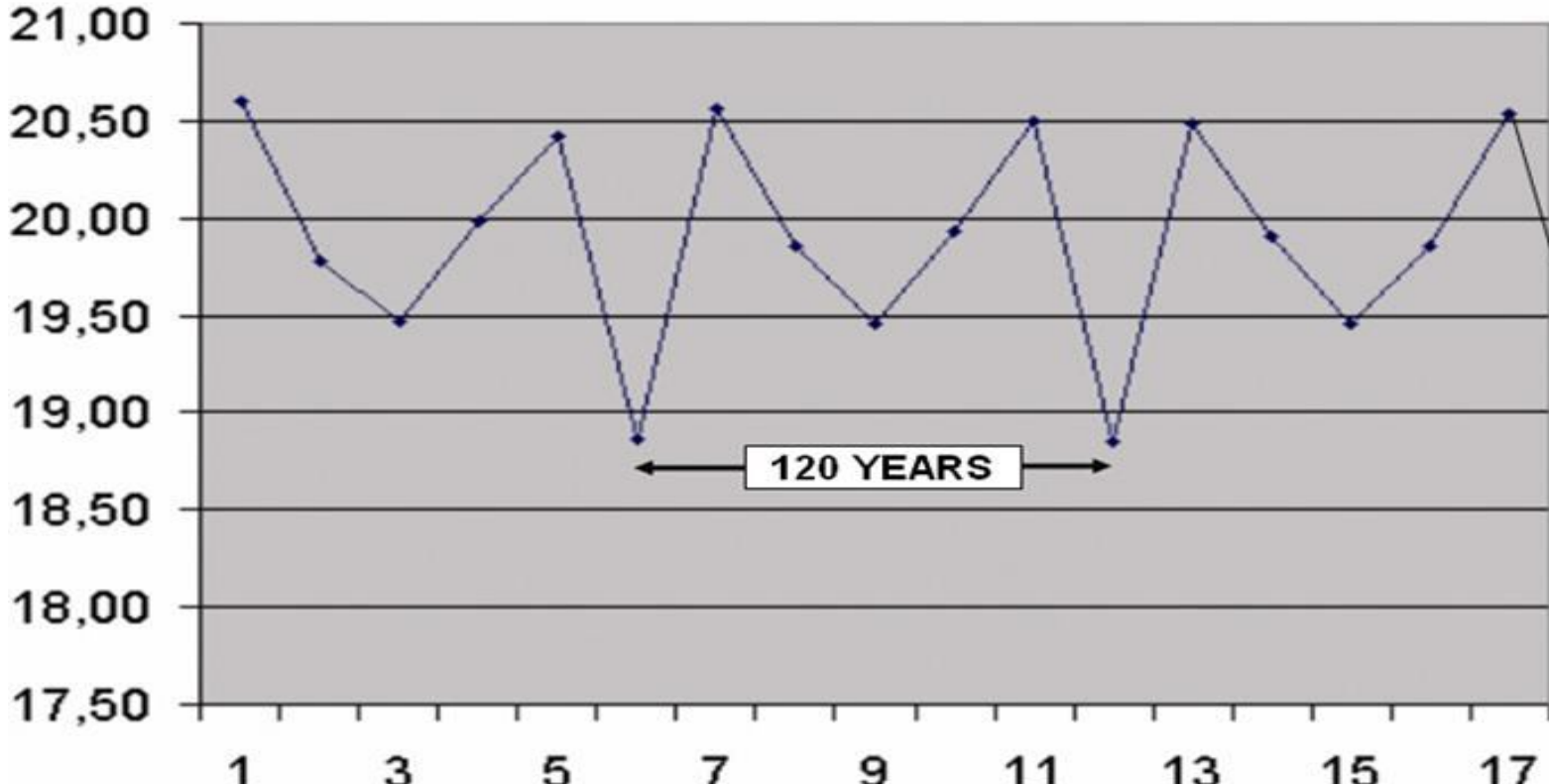

Figure 13. The above graph has been created with the program Chart Wizard of Microsoft Excel by applying the synodic period of Jupiter-Saturn as derived from the base of Redshift5. As horizontal axis $X$, I took 17 random consecutive conjunctions and as vertical axis $Y$, I took the corresponding value of the synodic period of Jupiter \& Saturn-which as it is known-oscillates from 18.8 to 20.6 years. One can easily observe that the oscillation of a simple conjunction is a periodic phenomenon repeated every 120 years. Please note the clear formation of the letter " $M$ ", the first letter of the word Messiah! Even when, in the table with the simple conjunctions of Jupiter-Saturn, we insert a triple one, and if we consider only the date of the first conjunction and ignore the second and the third consecutive ones, then the graph continues to show infinitively the letter " $M$ ". 


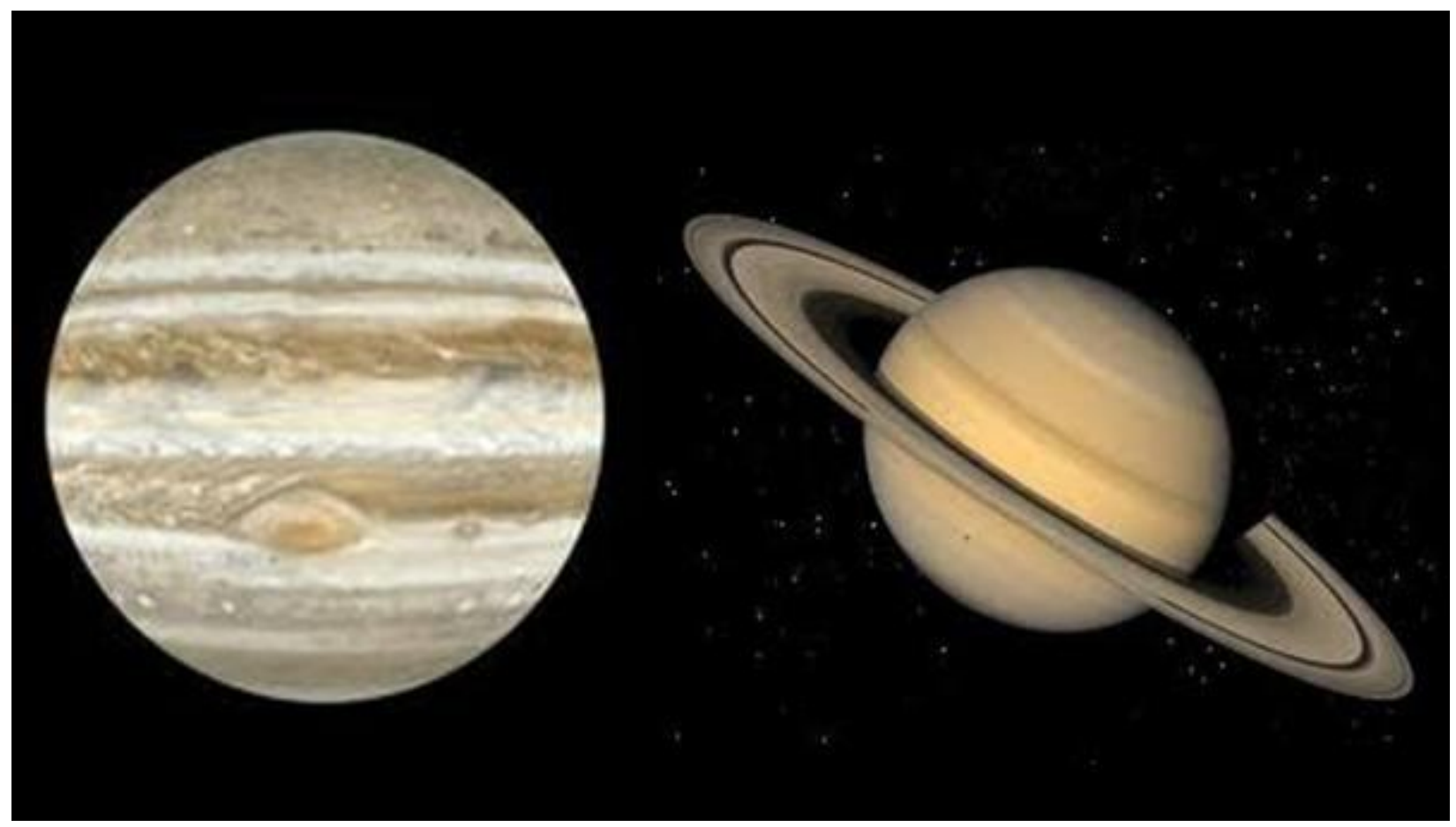

The giant planets Jupiter \& Saturn: Their conjunction in 7 B.C. was the Star of Bethlehem.

Citation: Sofias Sotirios. "REVEALING THE STAR OF BETHLEHEM"

Copyright: (C) 2021 Sofias Sotirios. This is an open-access article which permits unrestricted use, distribution, and reproduction in any medium, provided the original author and source are credited.

\section{References}

- Bethlehem Star Decoded by S.Sofias, Noon publications 2009

- The planetary programs Redshift 5, Maris Technologies, and Celestron's The Sky,

- The star of Christmas era, D. Simopoulos

- The Gospels, Matthew and Luke

- The Old Testament

- The Encarta Atlas of the World, Microsoft

- Article by D. Simopoulos in the Discovery magazine, January 2006

- Article by D.Theodossis in the Third Eye magazine, January 2006

- Desktop diary

- The world web 